\title{
Review
}

\section{New Targeted Therapies and Combinations of Treatments for Cervical, Endometrial and Ovarian Cancers: A Year in Review}

\author{
Adelina Silvana Dragomir 1,+,*, Elena Adriana Dumitrescu ${ }^{1,+}$, Isabela Anda Komporaly ${ }^{1}$, Raluca Ioana Mihăilă ${ }^{1}$, \\ Cristian Virgil Lungulescu ${ }^{2}$, Dana Lucia Stănculeanu ${ }^{1}$ \\ 1 Department of Oncology, “Carol Davila” University of Medicine and Pharmacy, 020021 Bucharest, Roma- \\ nia; adelina-silvana.gheorghe@drd.umfcd.ro (A.S.D.); elena-adriana.dumitrescu@drd.umfcd.ro (E.A.D.); \\ isabela-anda.komporaly@drd.umfcd.ro (I.A.K.); raluca-ioana.mihaila@drd.umfcd.ro (R.I.M); dana.stan- \\ culeanu@umfcd.ro (D.L.S.) \\ 2 Department of Medical Oncology, University of Medicine and Pharmacy Craiova, 200349 Craiova, Roma- \\ nia; cristilungulescu@yahoo.com \\ * Correspondence: adelina-silvana.gheorghe@drd.umfcd.ro \\ + These authors contributed equally to this work.
}

\begin{abstract}
This review of the meaningful data from 2021 on cervical, endometrial, and ovarian cancers aims to provide an update of the most clinically relevant studies presented at important oncologic congresses during the year [the American Society of Clinical Oncology (ASCO) Annual Meeting, the European Society for Medical Oncology (ESMO) Congress and the Society of Gynecologic Oncology (SGO) Annual Meeting]. Despite the underlying existence of the COVID-19 pandemic, the last year has been notable in terms of research, with significant and promising advances in gynecologic malignancies. Several major studies reporting the effects of innovative therapies for patients with cervical, endometrial, and ovarian cancers might change the medical practice in the future.
\end{abstract}

Keywords: cervical cancer, endometrial cancer, ovarian cancer, 2021 update, novel targeted therapies, immunotherapy

\section{Introduction}

Recent preclinical and clinical research has led to impressive advances in genital cancer, from examining its cellular origins to obtaining an outlook into the mechanisms of DNA damage repair that can be used for various therapies. Moreover, studies have shown clinical benefits for inhibition of PARP (poly (ADP-ribose) polymerase) and cell cycle modulation and have identified molecular features related to the therapeutic response.

In 2020, the COVID-19 pandemic has dominated the medical world, too, leading public health policies and scientific research efforts. Nevertheless, for women whose lives are affected by gynecological cancers (mainly cervical, uterine, and ovarian cancers), the impact of these neoplasms on incidence and mortality has been taken into account. Thus, clinical care and research have been forced to adapt in response to the pandemic, and encouragingly, research in gynecological cancers has remained active.

\section{Cervical cancer}

Cervical cancer remains one of the most common diagnoses of cancer in women, despite the spread of screening programs. Worldwide, it has a higher incidence and mortality rate than uterine and ovarian cancer, according to Globocan 2020 data, being on the fourth place in incidence in women (604,127 cases detected in 2020, cumulative risk of $1.8 \%)$, after breast, colorectal, and lung cancer. (1) 


\subsection{Screening and prevention}

The World Health Organization developed and published in July 2021 the second edition of the guide for screening and treatment of pre-cancerous cervical lesions for the prevention of cervical cancer. (2) In addition to vaccination against human papillomavirus (HPV - Human papillomavirus), the main etiological factor for the development of cervical cancer, the implementation of this global screening strategy could prevent more than 62 million deaths caused by cervical cancer in the next 100 years. WHO recommends HPV DNA detection in a "screening, triage and treatment" approach from the age of 30, with regular screening every 5-10 years, for the general population and from the age of 25 , with regular screening every 3 to 5 years, for the HIV-positive population. A recently published study showed that the COVID-19 pandemic dramatically reduced (by 82-92\%) cervical cancer screening in the United States between January and June 2020. (3)

The results of a recent genomic-scale association study provide new evidence of genetic susceptibility to cervical cancer. The results of a recent genomic-scale association study provide new evidence of genetic susceptibility to cervical cancer, especially gene variants of PAX8, CLPTM1L, and HLA, suggesting that their mutations disrupt the pathways of apoptotic and immune functions. Future studies that integrate the interaction between the host and the virus, genetics, and epigenetics, could further elucidate the complex interactions that predispose to cervical cancer. (4)

\subsection{Surgery}

Regarding surgery, minimally invasive radical hysterectomy has been associated with lower rates of disease-free survival and OS than open abdominal radical hysterectomy in women with early-stage cervical cancer, according to the LACC study (The Laparoscopic Approach to Cervical Cancer). (5) After the publication of this study, a recent assessment shows that the use of minimally invasive surgery decreased by $73 \%$ in academic centers and by $19 \%$ in non-academic centers $(p=0.004)$. (6)

\subsection{Radiotherapy, chemotherapy, and brachytherapy}

For locally advanced cervical cancer, radiotherapy concomitant with chemotherapy (cisplatin) and brachytherapy have been the standard of care since 1999. However, many patients relapse, and, in many cases, distant metastases occur. Over time, various studies have suggested that more adjuvant chemotherapy after concomitant radio-chemotherapy could bring additional benefits. Despite the flaws from these studies - including short follow-up and treatment intolerance, they have changed the practice in some centers.

International Phase III OUTBACK study tested the effect of four cycles of adjuvant chemotherapy (carboplatin AUC5 and paclitaxel at $155 \mathrm{mg} / \mathrm{m} 2$ ) after concomitant radiochemotherapy in women with locally advanced disease (FIGO stages IB1 with positive lymph nodes, IB2, II, IIIB or IVA), the primary goal being overall survival (OS) at 5 years. (7) After a median follow-up of 5 years, OS at 5 years was $71 \%$ in the group with radiochemotherapy and $72 \%$ in the one with the addition of adjuvant chemotherapy ( $\mathrm{HR}=0.90$; $\mathrm{P}=0.8$ ). The progression-free survival of the disease (PFS) was also similar between arms: $61 \%$ and respectively $63 \%(\mathrm{HR}=0.86 ; \mathrm{P}=0.6)$. The study concluded that in women with locally advanced cervical cancer, the adjuvant chemotherapy does not add any benefit to standard concomitant radio-chemotherapy based on cisplatin, as reported at the annual ASCO 2021 conference. In the phase III INTERLACE study, which is still ongoing, additional induction chemotherapy is evaluated before radio-chemotherapy, which may induce a better response and increased tolerance from the patients. (8)

The chemotherapy and the image-guided adaptive brachytherapy (IGABT) based on MRI (magnetic resonance imaging) resulted in effective and long-term stable local control at all stages of locally advanced cervical cancer with tolerable side effects. These results published in 2021 (prospective EMBRACE-I cohort study) is a positive discovery in the treatment of locally advanced cervical cancer, which could be used as a benchmark for 
clinical practice and all future studies. At a median follow-up of 51 months, overall 5-year disease control was $92 \%$, being different depending on the FIGO stage: it ranged from $89 \%$ in IIA2 and IVB to $98 \%$ in IB1 and $100 \%$ in IIIA. (9)

The final analysis of the PARCER study showed that survival without gastrointestinal toxicity of grade $\geq 2$ improved by $78 \%$ with radiotherapy with image-guided intensitymodulated radiotherapy (IG-IMRT) at the end of 3 years compared to $57 \%$ with 3D conformal radiotherapy, while survival without toxicity grade $\geq 3$ was $98 \%$ after IG-IMRT compared to $82 \%$ after 3D radiotherapy. (10) Furthermore, the data showed a significant reduction in acute diarrhea among patients receiving IG-IMRT vs. 3D radiotherapy.

Endostar, the recombinant human (rh)-endostatin (a fragment derived from type XVIII collagen) with anti-angiogenic properties, was analyzed in combination with platinum-based chemotherapy in the first-line treatment of recurrent/metastatic cervical cancer, through a single-arm, prospective phase II study. With a median PFS of 12 months, an overall response rate of $50.0 \%$, and a disease control rate of $71.4 \%$, the combination of platinum-based chemotherapy and endostar resulted to have a high level of effectiveness.

\subsection{Immunotherapy}

Encouraging data have become clear for checkpoint inhibitors as a second-line treatment for recurrent disease. The PD-L1 inhibitor cemiplimab-rwlc became the first immunotherapist to produce a statistically and clinically significant survival benefit in recurrent or metastatic cervical cancer that progressed after first-line platinum-based chemotherapy. Second-line treatment with cemiplimab resulted in a $27 \%$ decrease in the risk of death from chemotherapy in the squamous cell carcinoma population in the global phase III randomized study EMPOWER-Cervical 1/GOG-3016/ENGOT-cx9. The median OS in this group was 11.1 months with cemiplimab compared with 8.8 months with chemotherapy $(\mathrm{HR}=0.73 ; \mathrm{P}=0.00306)$. (12)

FDA (The Food and Drug Administration) has accepted a license application for accelerated approval (application for permission to place a biological product on the market) for balstilimab, an anti-PD-1 antibody, for the treatment of patients with recurrent or metastatic cervical cancer with progression of disease during or after chemotherapy. Balstilimab is a fully-humanized G4 monoclonal immunoglobulin (IgG4), designed to block PD1 interaction with its ligands, PD-L1 and PD-L2. (13)

Balstilimab is currently being investigated in clinical trials as monotherapy and in combination with the anti-CTLA-4 antibody zalifrelimab. The findings of a large (155 patients) single-arm phase II study evaluating the safety and antitumor activity of balstili$\mathrm{mab} 3 \mathrm{mg} / \mathrm{kg}$ Q2W in combination with zalifrelimab $1 \mathrm{mg} / \mathrm{kg}$ Q6W for up to 2 years in previously treated patients with recurrent/metastatic cervical cancer, showed impressive response rates (including complete remissions - 8.8\%), duration of response (9.3 months not reached) and OS (69\% at 6 months and $52.7 \%$ at 12 months), with manageable tolerability. The clinical benefit was highest in patients with PD-L1 positive tumors, but activity was present also in PD-L1 negative tumors. (14)

Regardless of PD-L1 expression or concurrent bevacizumab usage, pembrolizumab plus chemotherapy improved PFS and OS in patients with persistent, recurrent, or metastatic cervical cancer, according to the randomized, double-blind, phase III KEYNOTE826 study, presented at ESMO 2021 as the first interim analysis. These findings show that pembrolizumab plus chemotherapy, with or without bevacizumab may be a new standard of care for this population, with a tolerable safety profile. (15)

In patients with recurrent/advanced cervical cancer, toripalimab (a humanized IgG4 antibody specific for human PD-1 receptor), in combination with concurrent chemoradiotherapy showed promising anti-tumor effectiveness in a retrospective study presented at ESMO 2021: out of 25 patients included, 23 patients had objective responses (16 complete responses and 7 partial responses), with a 6 -month duration of response rate of $92 \%$. 
Moreover, toripalimab had a tolerable safety profile, suggesting that it might be a potential therapeutic option for this population. (16)

Tremelimumab (fully human monoclonal antibody against CTLA-4) plus durvalumab (anti-PD-L1 antibody) combined with metronomic oral vinorelbine in recurrent cervical cancer was investigated in the multi-cohort phase I/II MOVIE trial. Phase II of the study met its primary endpoint, the clinical benefit rate: objective response rate was $41.4 \%$ with 5 complete responses, 7 partial responses, and 4 stable diseases $\geq 24$ weeks. There is required further research for the combination of chemotherapy and immunotherapy in this group of patients. (17)

SHR-1701, a new bifunctional fusion protein comprised of a monoclonal antibody against PD-L1 linked to the extracellular domain of TGF- $\beta$ receptor II, was evaluated in a phase I study for patients with advanced cervical cancer who had progressed on 1 or 2 lines of platinum-based therapy (or were intolerant to it). Even though the median PFS was only 1.8 months, SHR-1701 holds promising antitumor activity and may prove to be a treatment option after further research. (18)

For many cancers, the combination of antiangiogenic therapy and immune checkpoint inhibitors has emerged as a viable treatment option. A phase II study was carried out to determine if anlotinib (a new multi-target tyrosine kinase inhibitor) combined with sintilimab (a PD-1 antibody) can improve the effectiveness and safety of patients with advanced cervical cancer. In the cohort of 42 patients enrolled, the overall response rate was $61.5 \%$ and the disease control rate was $94.9 \%$, with a median PFS of 9.4 months, providing a good perspective of this treatment (anlotinib orally $10 \mathrm{mg}$, qd, days 1-14 of a cycle of 21 days and sintilimab intravenously 200mg, q3w). (19)

Camrelizumab (immune checkpoint inhibitor, anti-PD-1 antibody), apatinib/rivoceranib (tyrosine kinase inhibitor, blocker of vascular endothelial growth factor receptor-2), and albumin-bound paclitaxel (nab-paclitaxel) were assessed in advanced cervical cancer, proving a good interaction in terms of effectiveness, with manageable adverse reactions: overall response rate was $71 \%$, with 5 complete responses, median PFS was 15.0 months, while the median duration of response and median OS were not reached. (20)

\subsection{Antibody-drug conjugates and vaccines}

In a recently published phase II study (innovaTV 204/GOG-3023/ENGOT-cx6), the authors found that tisotumab vedotin (an antibody-drug conjugate) produced lasting responses in patients previously treated with recurrent or metastatic cervical cancer. (21) In the study, 101 patients with recurrent or metastatic cervical cancer (squamous cell, adenocarcinoma, or adenosquamous) were enrolled between June 2018 and April 2019. The patients had progressive disease during or after chemotherapy with bevacizumab and had previously received no more than two systemic treatment regimens for recurrent or metastatic disease. Treatment consisted of intravenous tisotumab vedotin $(2 \mathrm{mg} / \mathrm{kg}$, up to a maximum of $200 \mathrm{mg}$ ) once every 3 weeks until disease progression or unacceptable toxicity. The primary goal was the objective response rate, with a median follow-up at the time of the 10-month analysis. Objective response was observed in 24 patients $(24 \%$, $95 \% \mathrm{CI}=16 \%-33 \%)$, including complete response in $7(7 \%)$. Another 49 patients $(49 \%)$ had stable disease, resulting in a disease control rate of $72 \%$. The average duration of response was 8.3 months, and median PFS was 4.2 months and median OS was 12.1 months. (21)

ENGOT-Cx8/GOG-3024/innovaTV 205 study, reported as interim results at ESMO 2021, showed that both first-line tisotumab vedotin + carboplatin ( $55 \%$ objective response rate, $6 \%$ complete responses and $48 \%$ partial responses, median PFS of 6.9 months) and second/third-line tisotumab vedotin + pembrolizumab (35\% objective response rate, $6 \%$ complete responses and $29 \%$ partial responses, median PFS of 5.6 months) had a promising antitumor activity with acceptable safety profiles in patients with recurrent or metastatic cervical cancer. (22) 
Interim results of a Korean phase II study indicated the effectiveness of the combination of pembrolizumab with the GX-188E therapeutic DNA vaccine (tirvalimogen teraplasmid) in patients with advanced cervical cancer, HPV-16 or HPV-18 positive. The combination of pembrolizumab and GX-188E (which induces HPV E6- and E7-specific Tcell activation) had a response to $42 \%$ of the patients evaluated; $15 \%$ had a complete response and $27 \%$ had a partial response. Treatment-related adverse events were easily manageable. (23)

\subsection{Targeted therapy}

BUL719 (alpelisib) has been used in the treatment of PIK3CA-mutated advanced/recurrent cervical cancer when at least two lines of therapy have failed, in a small study from Istituto Nazionale dei Tumori (Milano, Italy). For the 6 patients included, the objective response rate was $33 \%$, but the disease control rate was $100 \%$, with a mean duration of response of 6.6 months (two patients had partial response and four patients had stable disease). More research is needed to determine alpelisib's role in terms of efficacy and safety in PIK3CA-mutated advanced/recurrent cervical cancer. (24)

\section{Endometrial cancer}

Cervical cancer ranks sixth in incidence in women worldwide, according to GLOBOCAN 2020 data, with 417,367 new cases in 2020 and cumulative risk of 1.6\%. (1)

\subsection{Surgery}

Although primary debulking surgery is often considered standard for the treatment of stage IV endometrial cancer, this is associated with significant morbidity and low survival. Neoadjuvant chemotherapy has been proposed as an alternative treatment strategy. In a cohort study of 4890 women with metastatic endometrial cancer, 952 women (19.5\%) were treated with neoadjuvant chemotherapy. Survival for women treated with neoadjuvant chemotherapy was superior to that of women treated with primary debulking surgery for 3 to 8 months after initiation of treatment, after which survival was superior for those treated with primary debulking surgery. These findings suggest that women treated with primary debulking surgery have an increased risk of early death, but have a more favorable long-term prognosis. (25)

\subsection{Immunotherapy \pm targeted therapy}

On July 21, 2021, FDA approved pembrolizumab in combination with lenvatinib for patients with advanced endometrial carcinoma who do not have microsatellite instabilityhigh (MSI-H) or mismatch repair deficiency of DNA (dMMR), according to the results of the KEYNOTE-775 Study/Study 309. These patients must have progressive disease after any previous systemic therapy and should not be candidates for curative surgery or radiation therapy. (26) For patients with advanced endometrial cancer other than MSI-H or dMMR, the median PFS was 6.6 months $(95 \% \mathrm{CI}=5.6-7.4$ months $)$ in patients receiving pembrolizumab/lenvatinib and 3.8 months $(95 \% \mathrm{CI}=3.6-5.0$ months) for those receiving chemotherapy of the investigator's choice $(\mathrm{HR}=0.60,95 \% \mathrm{CI}=0.50-0.72, \mathrm{p}<0.0001)$. The average OS was 17.4 months $(95 \% \mathrm{CI}=14.2-19.9$ months $)$ and respectively 12.0 months (95\%CI $=10.8-13.3$ months) $-\mathrm{HR}=0.68,95 \% \mathrm{CI}=0.56-0.84, \mathrm{p}=0.0001)$. Objective response rate was 30\% (95\%CI $=26 \%-36 \%)$ and respectively $15 \%(95 \% \mathrm{CI}=12 \%-19 \%), \mathrm{p}<0.0001)$. The average duration of the response was 9.2 months, respectively 5.7 months in the 2 arms. (26)

In addition, the FDA accepted a new application for an additional license for review, seeking the approval of pembrolizumab monotherapy for the treatment of patients with advanced endometrial carcinoma MSI-H or dMMR, after the progression of the disease following any previous systemic therapy and who are not candidates for curative surgery or radiation therapy. (27) The application is based on the general response data of the 
KEYNOTE-158 study, presented at ESMO 2021. Pembrolizumab proved a durable overall response rate $(48 \%)$, with $14 \%$ complete responses, improving survival in patients with advanced MSI-H or dMMR endometrial cancer, heavily pretreated. This monotherapy also had manageable treatment-related adverse events. (28)

PD-1 inhibitor dostarlimab has been granted by FDA the accelerated approval for treatment of adult patients with recurrent or advanced endometrial cancer, which progressed during or after a previous platinum-based therapeutic regimen. (29) Phase I GARNET study showed an objective response rate of $42.3 \%(95 \% \mathrm{CI}=30.6 \%-54,6 \%)$, with a complete response in $12.7 \%$ of patients. At a median follow-up of 14.1 months, the mean duration of response was not reached, $93.3 \%$ of responses being preserved for at least 6 months. (30) The recommended dose of dostarlimab is $500 \mathrm{mg}$ given as a 30-minute intravenous infusion every 3 weeks for doses of one to four, and subsequent dosing begins 3 weeks after the fourth dose (1000 mg, 30-minute intravenous infusion every 6 weeks).

The combination of immunotherapy and targeted therapy has been assessed also in endometrial cancer. Anlotinib (novel oral tyrosine kinase inhibitor targeting c-kit, fibroblast growth factor receptor, platelet-derived growth factor receptors, and vascular endothelial growth factor receptor) plus sintilimab (anti-PD-1 immunoglobulin G4 monoclonal antibody) was studied in a prospective open-label, single-arm, phase II clinical trial, in patients with recurrent advanced endometrial cancer. The overall response rate was $77.3 \%$, with a disease control rate of $86.4 \%$ and a median PFS of 4.8 months, showing a promising treatment alternative after more research in the future. (31)

\section{Ovarian cancer}

In June 2021, ASCO has published a guide based on resources, which provides evidence-based recommendations for the evaluation of women with ovarian masses, as well as guidance on the treatment of epithelial ovarian cancer in regions that do not have adequate resources to provide high-level care. (32) Assessment of symptomatic adult women includes assessment of symptoms, family history, abdominopelvic ultrasound, and dosing of serum tumor marker CA-125, where possible. Additional imaging is recommended if CT/MRI resources are available. Diagnosis, staging, and/or treatment involve primarily surgery, before which it is necessary to investigate the presence of metastases. Treatment requires histological confirmation; the surgical goal is to stage the disease and perform complete cytoreduction until the absence of residual disease. In first-line therapy, platinum-based chemotherapy is recommended; in advanced stages, patients may receive neoadjuvant chemotherapy. After neoadjuvant chemotherapy, all patients should be assessed for interval debulking surgery (interval debulking surgery). Targeted therapy is not recommended in environments/countries with limited medical conditions. Specialized interventions are resource-dependent, for example, laparoscopy, fertility preservation surgery, genetic testing, and targeted therapy. Multidisciplinary care for ovarian cancer and palliative care should be provided, regardless of the environment or resources.

\subsection{Surgery}

FDA authorized on November 29, 2021 an adjuvant for the interoperative detection of malignant lesions in adult patients with ovarian cancer. Pafolacianine sodium injection (OTL38) is a fluorescent medication that operates by targeting the folate receptor, which is overexpressed in ovarian cancer, with the aid of near-infrared fluorescence (NIRF) imaging. The main objective was to achieve R0, which is known to be the strongest predictor of overall survival and was supported by the results of a single-arm, multicenter, openlabel trial (NCT03180307), in which NIRF imaging with pafolacianine sodium identified extra lesions that were not scheduled for excision and were not discovered by standard white light or palpation in $33 \%$ of patients (36 of 109). (33)

\subsection{Chemotherapy and Anti-angiogenic treatment}


The first-line therapeutic standard for epithelial ovarian cancer remained the combination of paclitaxel and carboplatin, along with cytoreductive surgery. Maintenance with Bevacizumab has been approved since 2016, and more recently, "front-line" maintenance treatment with PARP inhibitors has become the standard of care in ovarian cancer.

The studies GOG-218 and ICON7/AGO-OVAR 11 showed that early and continuous addition of bevacizumab for 15 months and respectively 12 months to the carboplatin / paclitaxel standard, respectively, significantly improved the PFS of the disease. In both studies, the maximum benefit was seen at the time of the highest cumulative exposure of bevacizumab - immediately after the last cycle of bevacizumab. (34) Nonetheless, the optimal duration of bevacizumab has never been clearly established, so the recent randomized phase III ENGOT/GCIG study examined whether prolonging bevacizumab treatment up to 30 months would improve its efficiency. (35) Treatment with bevacizumab for a longer period of time did not improve either PFS or OS in patients with epithelial ovarian cancer, fallopian tubes, or primary peritoneal cancer.

Adding bevacizumab to ixabepilone (azaepothilone B), a microtubule stabilizer, could be a promising treatment strategy for a group of platinum-resistant or refractory ovarian cancer patients, who currently lack a wide range of treatment options, according to data presented at the virtual edition of the annual meeting of the Society of Gynecological Oncology (SGO) 2021. The combination of bevacizumab plus ixabepilone significantly improved the objective response rate, PFS and OS compared to ixabepilone alone. The results of the randomized phase II study showed that $33 \%$ of patients responded to bevacizumab plus ixabepilone compared to $8 \%$ of those receiving ixabepilone alone, and median PFS doubled with the combination (5.5 vs. 2.2 months; $\mathrm{HR}=0.33$ ). (36)

\subsection{Antibody-Drug Conjugates}

In patients with recurrent ovarian cancer, the antibody-drug conjugate mirvetuximab soravtansine co-administered with bevacizumab, has shown anti-tumor activity that leads to lasting responses in platinum "agnostic" cases (resistant/sensitive), with strong folate receptor alpha expression (FR- $\alpha$ ). (37) The combination led to a response rate of $64 \%$, a mean response time of 11.8 months, and a median PFS of 10.6 months in patients with high FR- $\alpha$ expression in the Phase I study FORWARD II. (38)

The FDA approved the accelerated review for STRO-002 in August 2021, an antibody-drug conjugate anti-FR- $\alpha$, for the treatment of patients with epithelial ovarian cancer, fallopian tubes, or primary peritoneal cancer resistant to platinum, who have received one to three previous lines of systemic therapy, according to data from phase I study STRO-001-GM1. (39)

\subsection{Immunotherapy}

Phase III study IMagyn050/GOG 3015/ENGOT-OV39 showed that the addition of atezolizumab to bevacizumab and chemotherapy did not significantly improve PFS in newly diagnosed stage III or IV ovarian cancer patients, neither among all patients nor among those with positive PD-L1 expression. (40)

Phase III study "JAVELIN Ovarian 100" (NCT02718417) was suspended because it has not shown any benefit of PFS to the concomitant addition of avelumab and/or as a chemotherapy maintenance treatment (carboplatin/paclitaxel) in patients previously untreated with advanced epithelial ovarian cancer. (41) In addition, the phase III study "JAVELIN Ovarian 200" has not shown any significant improvement in PFS or OS with avelumab alone or in combination with pegylated liposomal doxorubicin (PLD) vs. PLD alone in patients with flat or refractory ovarian cancer. (42)

Immunotherapy has been studied also in the neoadjuvant setting for unresectable stage IIIC/IV ovarian cancer, in the phase Ib INEOV trial. It was proved to be feasible and safe for the administration of neoadjuvant durvalumab +/- tremelimumab with 
carboplatin and paclitaxel, prior to interval debulking surgery. However, further research is required on this topic. (43)

\subsection{Targeted therapy}

In a phase II study, it was found that the addition of the oral inhibitor Wee1 kinase (adavosertib) to gemcitabine significantly improved PFS and OS in platinum-resistant or refractory patients, with recurrent high-grade serous ovarian cancer. PFS was longer with adavosertib plus gemcitabine ( 4.6 months $[95 \% \mathrm{CI}=3.6-6.4])$ vs. 3,0 months $(95 \% \mathrm{CI}=1.8$ 3.8) with placebo plus gemcitabine $-\mathrm{HR}=0.55,95 \% \mathrm{CI}=0.35-0.90 . \mathrm{p}=0.015)$. (44)

The novel multi-target tyrosine kinase inhibitor anlotinib was assessed for safety and effectiveness as monotherapy in patients with ovarian cancer that is recurrent or resistant, in a phase II prospective, single-arm, and single-center clinical study. For the 31 patients included, the median PFS was 5.32 months, while the median OS was not reached, with an overall response rate of $25.9 \%$. (45) Anlotinib was also studied also in combination with pemetrexed in patients with ovarian cancer resistant to platinum, in a single-arm, open-label, phase II study, showing a median PFS of 9.3 months ( $95 \% \mathrm{CI}=5.5$ 13.2). (46)

The interim results of a study designed as adding a plasmid encoding p62/SQSTM1 (a multi-domain protein that regulates inflammation, apoptosis, and autophagy) to the standard gemcitabine chemotherapy proved that it may be effective for patients with platinum-resistant ovarian cancer, resulting in a PFS of 5.7 months (compared to 2.4 months in the control group, $\mathrm{p}=0.08)$. (47)

Relacorilant, a selective glucocorticoid receptor modulator, is studied for its capacity to restore sensitivity to chemotherapy. In a tree-arm, randomized, open-label, phase II trial on patients with recurrent platinum-resistant ovarian cancer or platinum-refractory ovarian cancer, relacorilant was assessed in combination with nab-paclitaxel. The study demonstrated that an intermittent regimen of $150 \mathrm{mg}$ relacorilant the day before, of, and after the administration of $80 \mathrm{mg} / \mathrm{m} 2$ nab-paclitaxel (on days 1,8 and 15, of a 28-day cycle) led to an improved PFS and duration of response. (48)

\subsection{PARP inhibitors}

After the initial results were extremely positive, at the 5-year follow-up of the pivotal SOLO-1 study in women with newly diagnosed advanced ovarian cancer and BRCA1/2 mutation, the maintenance treatment with olaparib led to a doubling of the PFS, statistically significant, according to data presented at the SGO 2021 Annual Meeting. Median PFS for the general population was maintained well beyond the end of treatment: 56,0 months with olaparib versus 13.8 months with placebo $(\mathrm{HR}=0.33 ; 95 \% \mathrm{CI}=0.25-0.43)$. The 5-year PFS was $48 \%$ and $21 \%$, respectively. (49)

The Phase III SOLO2 / ENGOT-Ov21 study showed a numerically but statistically insignificant improvement, in the overall goal of survival with olaparib maintenance therapy compared to placebo in patients with recurrent platinum-sensitive ovarian cancer and BRCA1/2 mutation (51.7 months vs. 38.8 months). (50)

The randomized phase II trial OCTOVA aimed to compare olaparib with weekly paclitaxel and the combination of olaparib plus cediranib in recurrent ovarian cancer, either after previous PARP inhibitors administration, or anti-angiogenic treatment. The combination of olaparib + cediranib had a higher PFS compared to olaparib in monotherapy (HR=0.70; 60\%CI: $0.57,0.86 ; \mathrm{p}=0.08)$. However, there was no difference in terms of PFS between the cohorts that received olaparib and weekly paclitaxel (HR=0.97, 60\%CI: 0.79, 1.19; $\mathrm{p}=0.55)$. (51)

Addition of niraparib maintenance treatment after platinum-based first-line chemotherapy with bevacizumab has shown a clinical benefit in patients with advanced ovarian cancer, according to data from the OVARIO study, presented at SGO 2021. (52) The analysis of the Phase II study by OVARIO showed that $62 \%$ of patients in the general 
population were left without disease progression at 18 months, including $76 \%$ of patients in the homologous recombination deficit subgroup (HRD - homologous recombinationdeficient) and $47 \%$ of patients without homologous recombinant proficiency (HRP - homologous recombination-proficient). (53)

In patients with positive, advanced, relapsed BRCA ovarian cancer, the treatment with the PARP inhibitor rucaparib led to a significant improvement in PFS, compared to standard chemotherapy, according to the results of the international phase III study ARIEL4 (7.4 months vs. 5.7 months $-\mathrm{HR}=0.64, \mathrm{p}=0.001)$. (54)

A new PARP inhibitor may soon join the treatment of ovarian cancer, according to the data presented at SGO 2021. (55) The results of the phase III study (NCT03863860) of fuzuloparib (previously called fluzoparib) as maintenance therapy in patients with recurrent platinum-sensitive ovarian cancer showed a 7.4-month improvement in median PFS (12.9 vs 5.5 months; $\mathrm{P}<0.0001$ ) and a $75.5 \%$ reduced risk of disease progression or death compared to placebo $(\mathrm{HR}=0.25)$. (56)

In the ANNIE multicentre, single-arm, phase II trial, the safety and efficacy of niraparib (300mg/200mg once daily continuously) combined with anlotinib (12mg on day 1-14 of each 21-day cycle) was evaluated in patients with platinum-resistant recurrent ovarian epithelial, fallopian tube, or primary peritoneal cancer. The overall response rate was $50.0 \%$ and median PFS was not reached, therefore presenting antitumor activity that appears to be promising, but with hand-foot skin reaction as treatment-related side event (in $47.5 \%$ of patients). (57)

\section{Conclusions}

Despite the pandemic caused by COVID-19, the results presented here show the many therapeutic advances made in 2021 in the field of gynecological cancers (cervical, endometrial and ovarian). Table 1 summarizes the FDA approvals in gynecological cancers during 2021.

Table 1. FDA approvals in gynecological cancers during 2021

\begin{tabular}{|c|c|c|}
\hline Date & $\begin{array}{c}\text { Active ingredient } \\
\text { And } \\
\text { drug name }\end{array}$ & FDA-approved use \\
\hline April 22 & Dostarlimab-gxly (Jemperli) & Endometrial Cancer \\
\hline July 21 & $\begin{array}{l}\text { Pembrolizumab (Keytruda) } \\
\text { plus Lenvatinib (Lenvima) }\end{array}$ & $\begin{array}{c}\text { Advanced endometrial carcinoma that } \\
\text { is not MSI-H or dMMR. }\end{array}$ \\
\hline September 20 & Tisotumab Vedotin-tftv & $\begin{array}{c}\text { Recurrent or metastatic cervical cancer } \\
\text { who experienced disease progression } \\
\text { on or after chemotherapy. }\end{array}$ \\
\hline \multirow{2}{*}{ October 13} & $\begin{array}{l}\text { Pembrolizumab (Keytruda) } \\
\text { in combination with chemo- } \\
\text { therapy, with or without } \\
\text { bevacizumab (Avastin) }\end{array}$ & $\begin{array}{l}\text { Persistent, recurrent, or metastatic cer- } \\
\text { vical cancer whose tumors express } \\
\text { PD-L1 (combined positive score [CPS] } \\
\geq 1 \text { ), as determined by an FDA-ap- } \\
\text { proved test. }\end{array}$ \\
\hline & $\begin{array}{l}\text { Pembrolizumab (Keytruda) } \\
\text { as a single agent }\end{array}$ & $\begin{array}{l}\text { Recurrent or metastatic cervical cancer } \\
\text { with disease progression on or after } \\
\text { chemotherapy whose tumors express } \\
\text { PD-L1 (CPS } \geq 1 \text { ), as determined by an } \\
\text { FDA-approved test. }\end{array}$ \\
\hline November 29 & Pafolacianine (Cytalux) & $\begin{array}{l}\text { Ovarian cancer (to help identify can- } \\
\text { cerous lesions during surgery) }\end{array}$ \\
\hline
\end{tabular}


Translational research, focused on the results of preclinical studies, will further lead to the clinical integration of information obtained in the laboratory, in phase II and III studies, establishing an important basis and key research priorities for the future. By continuing the quest for best treatments by targeting novel and exploitable genetic and biologic abnormalities in cervical, endometrial, and ovarian malignancies, oncologists must be prepared to confront the challenge of achieving clinically substantial improvements in gynecologic oncology patients' outcomes.

Author Contributions: Conceptualization, A.S.D. and D.L.S.; methodology, A.S.D. and E.A.D.; resources, I.A.K.; data curation, R.I.M. and C.V.L.; writing-original draft preparation, A.S.D. and E.A.D.; writing - review and editing, D.L.S, I.A.K., R.I.M. and C.V.L; supervision, D.L.S.; project administration, A.S.D., E.A.M and D.L.S. All authors have read and agreed to the published version of the manuscript.

Funding: This research received no external funding.

Conflicts of Interest: The authors declare no conflict of interest.

\section{References}

1. Sung H, Ferlay J, Siegel RL, Laversanne M, Soerjomataram I, Jemal A, Bray F. Global cancer statistics 2020: GLOBOCAN estimates of incidence and mortality worldwide for 36 cancers in 185 countries. CA: a cancer journal for clinicians. 2021 May;71(3):209-49

2. World Health Organization. WHO guideline for screening and treatment of cervical pre-cancer lesions for cervical cancer prevention. 2021

3. DeGroff A, Miller J, Sharma K, et al. COVID-19 impact on screening test volume through the National Breast and Cervical Cancer early detection program, January-June 2020, in the United States. Preventive Medicine. 2021 Oct 1;151:106559

4. Bowden SJ, Bodinier B, Kalliala I, et al. Genetic variation in cervical preinvasive and invasive disease: a genome-wide association study. The Lancet Oncology. 2021 Apr 1;22(4):548-57

5. Ramirez PT, Frumovitz M, Pareja R, et al. Minimally invasive versus abdominal radical hysterectomy for cervical cancer. New England Journal of Medicine. 2018 Nov 15;379(20):1895-904

6. Lewicki PJ, Basourakos SP, Qiu Y, Hu JC, Sheyn D, Hijaz A, Shoag JE. Effect of a randomized, controlled trial on surgery for cervical cancer. New England Journal of Medicine. 2021 Apr 29;384(17):1669-71

7. Mileshkin LR, Narayan K, Moore KN, et al. A phase III trial of adjuvant chemotherapy following chemoradiation as primary treatment for locally advanced cervical cancer compared to chemoradiation alone: Outback. J Clin Oncol. 2021;39(suppl 15):abstr.LBA3

8. de Azevedo CR, Thuler LC, de Mello MJ, et al. Phase II trial of neoadjuvant chemotherapy followed by chemoradiation in locally advanced cervical cancer. Gynecologic oncology. 2017 Sep 1;146(3):560-5

9. Pötter R, Tanderup K, Schmid MP, et al. MRI-guided adaptive brachytherapy in locally advanced cervical cancer (EMBRACE-I): a multicentre prospective cohort study. The Lancet Oncology. 2021 Apr 1;22(4):538-47

10. Chopra S: Phase III randomized trial of postoperative adjuvant conventional radiation (3DCRT) versus image guided intensity modulated radiotherapy (IG-IMRT) in cervical cancer (PARCER): Final analysis. Society of Gynecologic Oncology Virtual Annual Meeting on Women's Cancer. Abstract ID \# 10224. 2021

11. Zhou J, Wang L, Zhuang H, Qin S, Xu X. 780P Safety and efficacy of endostar combined with platinum-based chemotherapy in the first-line treatment of recurrence and meta-static cervical cancer: A single-arm, prospective phase II study. Annals of Oncology. 2021 Sep 1;32:S754

12. Tewari KS, Monk BJ, Vergote I, et al. VP4-2021: EMPOWER-Cervical 1/GOG-3016/ENGOT-cx9: Interim analysis of phase III trial of cemiplimab vs. investigator's choice (IC) chemotherapy (chemo) in recurrent/metastatic (R/M) cervical carcinoma. Annals of Oncology. 2021 Jul 1;32(7):940-1

13. O'Malley DM, Oaknin A, Monk B, et al. Single-agent anti-PD-1 balstilimab or in combination with anti-CTLA-4 zalifrelimab for recurrent/metastatic (R/M) cervical cancer (CC): preliminary results of two independent phase II trials. Ann Oncol. 2020;31(suppl 4):S1164-S1165. doi:10.1016/j.annonc.2020.08.2264

14. O'Malley D, Neffa M, Monk BJ, et al. 724MO Balstilimab (anti-PD-1) in combination with zalifrelimab (anti-CTLA-4): Final results from a phase II study in patients (pts) with recurrent/metastatic (R/M) cervical cancer (CC). Annals of Oncology. 2021 Sep 1;32:S727

15. Colombo N, Dubot C, Lorusso D, et al. LBA2 Pembrolizumab plus chemotherapy versus placebo plus chemotherapy for persistent, recurrent, or metastatic cervical cancer: Randomized, double-blind, phase III KEYNOTE-826 study. Annals of Oncology. 2021 Sep 1;32:S1307-8

16. Song Y, Wang C, Li X, Li Y, Zhang C. 782P A retrospective study of toripalimab combined with concurrent chemoradiotherapy in patients with recurrent/advanced cervical cancer. Annals of Oncology. 2021 Sep 1;32:S755 
17. Frenel JS, Hervieu A, Borcoman E, et al. 775P Tremelimumab (T)+ durvalumab (D) combined with metronomic oral vinorelbine (MOV): Results of the recurrent cervical cancer (RCC) cohort of the MOVIE study. Annals of Oncology. 2021 Sep 1;32:S753

18. Feng J, Wang J, Tang D, et al. 776P SHR-1701, a bifunctional fusion protein targeting PD-L1 and TGF- $\beta$, for pretreated advanced cervical cancer: Data from a clinical expansion cohort of a phase I study. Annals of Oncology. 2021 Sep 1;32:S753

19. Xu Q, Chen C, Huang Z, et al. 774P Anlotinib plus sintilimab in patients with recurrent advanced cervical cancer: A prospective, multicenter, single-arm, phase II clinical trial. Annals of Oncology. 2021 Sep 1;32:S752-3

20. Li G, Zhao Y, Jiang Y, Yang Q, Huang A, Chen Y, Han D. 777P A prospective, single-arm, open-label study of camrelizumab, apatinib and nab-paclitaxel in patients with advanced cervical cancer. Annals of Oncology. 2021 Sep 1;32:S753-4

21. Coleman RL, Lorusso D, Gennigens C, et al. Efficacy and safety of tisotumab vedotin in previously treated recurrent or metastatic cervical cancer (innovaTV 204/GOG-3023/ENGOT-cx6): a multicentre, open-label, single-arm, phase 2 study. The Lancet Oncology. 2021 May 1;22(5):609-19

22. Vergote IB, Monk BJ, O'Cearbhaill RE, et al. 723MO Tisotumab vedotin (TV)+ carboplatin (Carbo) in first-line (1L) or+ pembrolizumab (Pembro) in previously treated (2L/3L) recurrent or metastatic cervical cancer ( $\mathrm{r} / \mathrm{mCC})$ : Interim results of ENGOT-Cx8/GOG-3024/innovaTV 205 study. Annals of Oncology. 2021 Sep 1;32:S726-7

23. Youn JW, Hur SY, Woo JW, Kim YM, Lim MC, Park SY, Seo SS, No JH, Kim BG, Lee JK, Shin SJ. Pembrolizumab plus GX188E therapeutic DNA vaccine in patients with HPV-16-positive or HPV-18-positive advanced cervical cancer: interim results of a single-arm, phase 2 trial. The Lancet Oncology. 2020 Dec 1;21(12):1653-60

24. Bogani G, Indini A, Bini M, Raspagliesi F. 778P BYL719 (alpelisib) for the treatment of PIK3CA-mutated, recurrent/advanced cervical cancer. Annals of Oncology. 2021 Sep 1;32:S754

25. Tobias CJ, Chen L, Melamed A, St Clair C, Khoury-Collado F, Tergas AI, Hou JY, Hur C, Ananth CV, Neugut AI, Hershman DL. Association of Neoadjuvant Chemotherapy with Overall Survival in women with metastatic endometrial Cancer. JAMA network open. 2020 Dec 1;3(12):e2028612

26. Makker V, Casado Herraez A, Aghajanian C, et al. A phase 3 trial evaluating efficacy and safety of lenvatinib in combination with pembrolizumab in patients with advanced endometrial cancer. Journal of Clinical Oncology. 2019:37;15_suppl

27. Valero C, Lee M, Hoen D, et al. Response Rates to Anti-PD-1 Immunotherapy in Microsatellite-Stable Solid Tumors with 10 or More Mutations per Megabase. JAMA oncology. 2021 May 1;7(5):739-43

28. O'Malley D, Bariani GM, Cassier PA, et al. 795MO Pembrolizumab (pembro) in patients (pts) with microsatellite instabilityhigh (MSI-H) advanced endometrial cancer (EC): Updated results from KEYNOTE-158. Annals of Oncology. 2021 Sep 1;32:S730-1

29. U.S. Food and Drug Administration: FDA grants accelerated approval to dostarlimab-gxly for dMMR endometrial cancer. Available at https://www.fda.gov/drugs/drug-approvals-and-databases/fda-grants-accelerated-approval-dostarlimabgxly-dmmr-endometrial-cancer. Accessed August 30, 2021

30. Oaknin A, Tinker AV, Gilbert L, et al: Clinical activity and safety of the anti-programmed death 1 monoclonal antibody dostarlimab for patients with recurrent or advanced mismatch repair-deficient endometrial cancer: A nonrandomized phase 1 clinical trial. JAMA Oncol 6:1766-1772, 2020

31. Wei W, Ban X, Yang F, Huang Y, Li J, Cheng X, Zheng M. 799P Anlotinib plus sintilimab in patients with recurrent advanced endometrial cancer: A prospective open-label, single-arm, phase II clinical trial. Annals of Oncology. 2021 Sep 1;32:S762

32. Vanderpuye VD, Clemenceau JR, Temin S, et al. Assessment of Adult Women With Ovarian Masses and Treatment of Epithelial Ovarian Cancer: ASCO Resource-Stratified Guideline. JCO Global Oncology. 2021 Jun;7:1032-66

33. Tanyi JL, Chon HS, Morgan MA, et al. Phase 3, randomized, single-dose, open-label study to investigate the safety and efficacy of pafolacianine sodium injection (OTL38) for intraoperative imaging of folate receptor positive ovarian cancer. Journal of Clinical Oncology. 2021;39:15_suppl,5503-5503

34. Perren TJ, Swart AM, Pfisterer J, Ledermann JA, Pujade-Lauraine E, Kristensen G, Carey MS, Beale P, Cervantes A, Kurzeder C, Bois AD. A phase 3 trial of bevacizumab in ovarian cancer. New England Journal of Medicine. 2011 Dec 29;365(26):2484-96

35. Pfisterer J, Joly F, Kristensen G, et al. Optimal treatment duration of bevacizumab combined with carboplatin and paclitaxel in patients with primary epithelial ovarian, fallopian tube, or peritoneal cancer. 2021 ASCO Annual Meeting. Abstract 5501. Presented June 7, 2021

36. Roque DM, Siegel ER, Buza N, et al: Randomized phase II trial of weekly ixabepilone with or without biweekly bevacizumab for platinum-resistant or refractory ovarian/fallopian tube/primary peritoneal cancer. SGO 2021 Annual Meeting on Women's Cancer. Abstract 11570. Presented March 25, 2021

37. O'Malley DM, Oaknin A, Matulonis UA, et al: Mirvetuximab soravtansine, a folate receptor alpha (FR $\alpha)$-targeting antibody-drug conjugate, in combination with bevacizumab in patients with platinum-agnostic ovarian cancer: Final analysis. 2021 ASCO Annual Meeting. Abstract 5504. Presented June 7, 2021

38. Moore K, Oza A, Colombo N, et al: FORWARD I (GOG 3011): A phase III study of mirvetuximab soravtansine, a folate receptor alpha (FRa)-targeting antibody-drug conjugate, versus chemotherapy in patients with platinum-resistant ovarian cancer. Ann Oncol. 2019;30(suppl 5):v403

39. Naumann RW, Uyar DS, Schilder RJ, et al. Abstract CT160: A Phase I, open-label, safety, pharmacokinetic, and preliminary efficacy study of STRO-002, an anti-folate receptor alpha (FolR $\alpha$ ) antibody drug conjugate (ADC), in patients with 
advanced epithelial ovarian cancer (including fallopian tube or primary peritoneal cancers) and endometrial cancers. Proceedings. 2019;79(suppl13)

40. Moore KN, Bookman M, Sehouli J, et al. Atezolizumab, bevacizumab, and chemotherapy for newly diagnosed stage III or IV ovarian cancer: placebo-controlled randomized phase III trial (IMagyn050/GOG 3015/ENGOT-OV39). Journal of Clinical Oncology. 2021 Jun 10;39(17):1842-55

41. Monk BJ, Colombo N, Oza AM, et al. Chemotherapy with or without avelumab followed by avelumab maintenance versus chemotherapy alone in patients with previously untreated epithelial ovarian cancer (JAVELIN Ovarian 100): an open-label, randomised, phase 3 trial. The Lancet Oncology. 2021 Aug 4

42. Pujade-Lauraine E, Fujiwara K, Ledermann JA, et al. Avelumab alone or in combination with chemotherapy versus chemotherapy alone in platinum-resistant or platinum-refractory ovarian cancer (JAVELIN Ovarian 200): an open-label, threearm, randomised, phase 3 study. The Lancet Oncology. 2021 Jun 15

43. Leary A, Rouge TD, Lortholary A, et al. 727P Phase Ib INEOV neoadjuvant trial of the anti-PDL1, durvalumab (D)+/-antiCTLA4 tremelimumab $(\mathrm{T})$ with platinum chemotherapy for patients (pts) with unresectable ovarian cancer (OC): A GINECO study. Annals of Oncology. 2021 Sep 1;32:S731

44. Lheureux S, Cristea MC, Bruce JP, et al. Adavosertib plus gemcitabine for platinum-resistant or platinum-refractory recurrent ovarian cancer: a double-blind, randomised, placebo-controlled, phase 2 trial. The Lancet. 2021 Jan 23;397(10271):28192

45. Wang H, Shan B, Shen W. 730P Anlotinib in patients with recurrent platinum-resistant or-refractory ovarian carcinoma: A prospective, single-arm, single-center, phase II clinical study. Annals of Oncology. 2021 Sep 1;32:S733

46. Chen J, Wei W, Zheng L, et al. 732P Anlotinib plus pemetrexed in patients with platinum-resistant ovarian cancer: A singlearm, open-label, phase II study. Annals of Oncology. 2021 Sep 1;32:S734

47. Polyakov S, Krasny S, Zhavrid E, et al. 759P Adding dosing of plasmid encoding p62/SQSTM1 to gemcitabine chemotherapy may provide clinical benefits to patients with platinum-resistant ovarian cancer. Annals of Oncology. 2021 Sep 1;32:S747-8

48. Colombo N, Nguyen DD, Fleming GF, et al. 7210 Relacorilant, a selective glucocorticoid receptor modulator, in combination with nab-paclitaxel improves progression-free survival in patients with recurrent platinum-resistant ovarian cancer: A 3-arm, randomized, open-label, phase II study. Annals of Oncology. 2021 Sep 1;32:S725

49. Bradley W, Moore K, Colombo N, et al: Maintenance olaparib for patients with newly diagnosed, advanced ovarian cancer and a BRCA mutation: 5-year follow-up from SOLO-1. Society of Gynecologic Oncology (SGO) 2021 Annual Meeting on Women's Cancer. Abstract 39. Presented March 20, 2021

50. Poveda A, Floquet A, Ledermann JA, et al. Olaparib tablets as maintenance therapy in patients with platinum-sensitive relapsed ovarian cancer and a BRCA1/2 mutation (SOLO2/ENGOT-Ov21): a final analysis of a double-blind, randomised, placebo-controlled, phase 3 trial. The Lancet Oncology. 2021 May 1;22(5):620-31

51. Nicum S, Holmes J, McGregor N, et al. $722 \mathrm{O}$ Randomised phase II trial of olaparib compared to weekly paclitaxel or olaparib plus cediranib in patients with platinum-resistant ovarian cancer (OCTOVA). Annals of Oncology. 2021 Sep 1;32:S725-6

52. Hardesty MM, Krivak T, Wright GS, et al: Phase 2 OVARIO study of niraparib plus bevacizumab therapy in advanced ovarian cancer following frontline platinum-base chemotherapy with bevacizumab. Society of Gynecologic Oncology Virtual Annual Meeting on Women's Cancer. Poster 22. Presented March 19, 2021

53. Mirza MR, „dqvist EA, Birrer MJ, et al. Niraparib plus bevacizumab versus niraparib alone for platinum-sensitive recurrent ovarian cancer (NSGO-AVANOVA2/ENGOT-ov24): a randomised, phase 2, superiority trial. The Lancet Oncology. 2019 Oct 1;20(10):1409-19

54. Kristeleit R, Lisyanskaya A, Fedenko A, et al: Rucaparib vs chemotherapy in patients with advanced, relapsed ovarian cancer and a deleterious BRCA mutation. Society of Gynecologic Oncology 2021 Virtual Annual Meeting on Women's Cancer. Abstract 1. Presented March 19, 2021.

55. Li N, Zhang Y, Wang J, et al: Fuzuloparib maintenance therapy in patients with platinum-sensitive, relapsed ovarian cancer: A multicenter, randomized, double-blind, placebo-controlled, phase III trial. Society of Gynecologic Oncology (SGO) 2021 Annual Meeting on Women's Cancer. Abstract 11557. Presented March 25, 2021

56. Li N, Bu H, Liu J, Zhu J, Zhou Q, Wang L, Yin R, Wu X, Yao S, Gu K, Zhang H. An Open-label, Multicenter, Single-arm, Phase II Study of Fluzoparib in Patients with Germline BRCA1/2 Mutation and Platinum-sensitive Recurrent Ovarian Cancer. Clinical Cancer Research. 2021 May 1;27(9):2452-8

57. Liu J, Liu G, Li J, Feng Y, Huang H, Wan T, Huang Q, Xian B, Kong B, Lin A. 736P Preliminary results of anlotinib and niraparib dual therapy evaluation in platinum-resistant recurrent ovarian cancer (ANNIE): A multicenter, single-arm, phase II trial. Annals of Oncology. 2021 Sep 1;32:S736 